\section{SIPE-Syndrom: Tod im kalten Wasser}

Das SIPE-Syndrom ist ein klassisches Lungenödem, dass bei jungen Sportlern, die längere Zeit im kalten Wasser schwimmen, ohne Vorwarnung auftreten kann. Typischerweise passiert dies beim Triathlon. Amerikanische Wissenschaftler untersuchten nun eine Möglichkeit zur Prävention dieses lebensgefährlichen Syndroms.

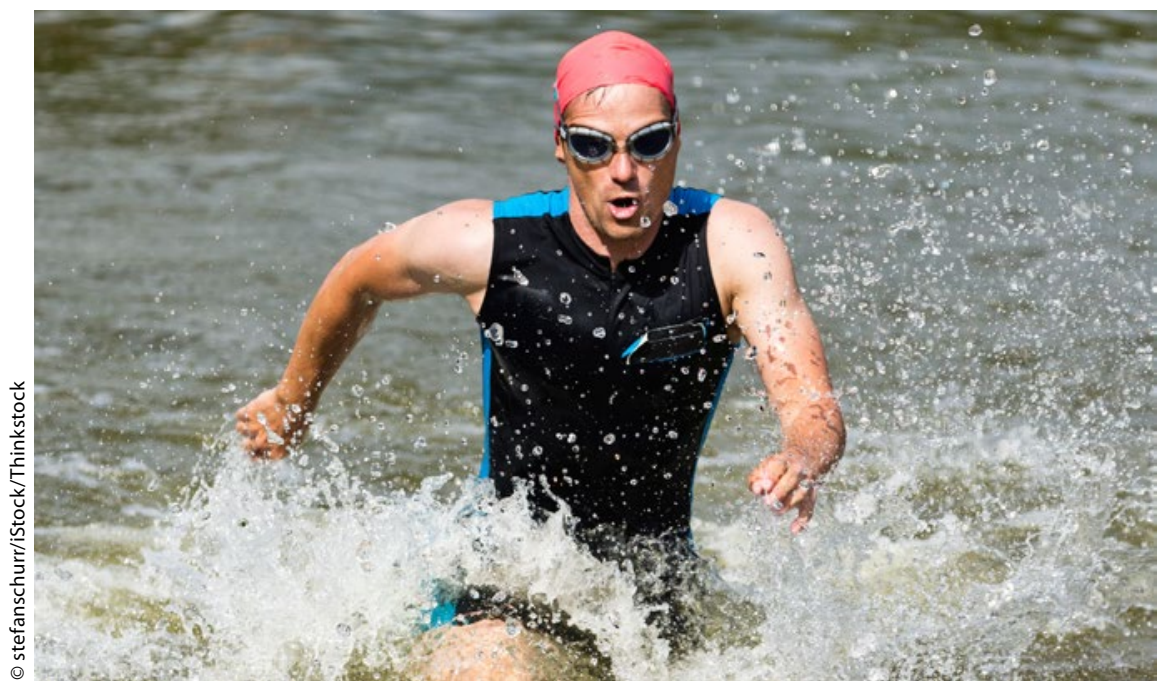

Risiko Triathlon: Das SIPE-Syndrom tritt bei 1-2\% der Triathleten auf.

mmer wieder wird berichtet, dass Triathleten während des Schwimmens eine plötzliche Lungenfunktionsstörung bekommen. 2015 starb ein Triathlet beim Schwimmen im Hudson River. Als Ursache wurde ein SIPE-Syndrom (Swimming-induced pulmonary edema) vermutet. Bei demselben Wettbewerb entwickelte eine Triathletin plötzlich beim Schwimmen schwere Atemnot nach einer Hustenattacke. Sie spuckte Blut. Innerhalb kurzer Zeit hatte sich ein Lungenödem entwickelt. Es wird vemutet, dass das SIPE-Syndrom bei 1-2\% aller Triathleten auftritt. Die American Heart Association berichtet von 43 Todesfällen beim Triathlon in den Jahren 2003 bis 2011, dabei traten 30 akute Todesfälle beim Schwimmwettbewerb auf.

Das Tückische dabei ist, dass sich diese Dyspnoe infolge eines Lungenödems innerhalb von wenigen Minuten entwickeln kann. Dramatische Folgen sind eine sich rasch entwickelnde Bewusstlosigkeit bis hin zum Herzstillstand. Meist kündigt sich dieses lebensgefährliche Syndrom in der Vorgeschichte nicht an.
Die Pathophysiologie des SIPE-Syndroms ist bis heute nicht vollständig geklärt. Sicher spielen die Zunahme des Pulmonalarteriendrucks und ein Stabilitätsverlust der Kapillarwand mit Wassereintritt in die Alveolen eine wichtige Rolle. Risikokondition sind kaltes Wasser, massive körperliche Belastung, erhöhte Flüssigkeitsaufnahme (Hyperhydrierung) und eine niedrige Vitalkapazität. Viele Betroffene haben zugrunde liegende kardiale Probleme, oft eine pathologische linksventrikuläre Funktion oder arterielle Hypertonie.

Gewöhnlich verschwinden die Symptome spontan innerhalb von 24 Stunden. Hilfreich sind betaadrenerge Agonisten (z. B. Salbutamol) und Diuretika sowie Steroide unter antibiotischer Abschirmung.

Forscher des Duke University Medical Center untersuchten zehn Sportler mit einem mittleren Alter von 41,6 Jahren, die in der Vorgeschichte ein SIPE-Syndrom entwickelten und 20 Kontrollpersonen (mittleres Alter 36,2 Jahre). Alle bekamen einen Katheter in die A. radia- lis und in die Pulmonalarterien eingelegt. Eine Belastung erfolgte in $20^{\circ} \mathrm{C}$ kaltem Wasser auf einem Fahrradergometer für einen Zeitraum von sechs bis sieben Minuten. Die Probanden wiederholten die Belastung 150 Minuten nach der Einnahme von $50 \mathrm{mg}$ Sildenafil.

Nach der Gabe von Sildenafil konnten keine signifikanten Unterschiede zwischen den beiden Gruppen in der Hämodynamik und auch keine Unterschiede bei der Belastbarkeit festgestellt werden. Die mittleren pulmonalarteriellen Drucke und die Wedge-Drucke waren gleich.

Die Ergebnisse dieser Studie bestätigen einerseits, dass SIPE eine besondere Form des Lungenödems ist, und andererseits, dass Sildenafil prophylaktisch wirksam ist hinsichtlich der Verhinderung eines SIPE-Syndroms.

Moon RE et al. Swimming-Induced Pulmonary Edema: Pathophysiology and Risk Reduction With Sildenafil. Circulation. 2016;133(10):988996.

\section{Kommentar}

Jeder Triathlet und Schwimmer im kalten Wasser sollte dieses schwere Krankheitsbild, das bei jedem ohne Vorwarnung auftreten kann, kennen - und wissen wie die Nofallbehandlung aussieht und was man prophylaktisch tun kann, um eine solche lebensbedrohliche Situation zu vermeiden. Hilfreich könnten Neoprenanzüge mit geringer Kompression sein. Veranstalter sollten darauf achten, dass das Wasser nicht zu kalt ist. Die Sportler sollten sich vor dem Beginn des Schwimmwettbewerbs langsam im Wasser akklimatisieren und sehr langsam beim Schwimmwettbewerb starten. Und last but not least sollten die Athleten bei den vorausgegangenen Wettbewerben nicht extrem viel getrunken haben. Salbutamol (max. 1.600 $\mu$ g pro 24 Stunden = 8-12 Hübe) gilt heute nicht mehr als Dopingmittel, die Einnahme muss aber vor dem Wettbewerb auf dem Dopingkontrollzettel gemeldet werden.

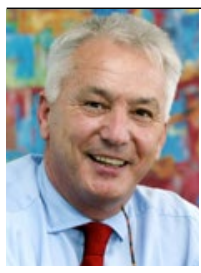

Prof. Dr. med. Curt Diehm

Max-Grundig-Klinik, Bühl 